\title{
Impact of breast-feeding and high- and low-protein formula on the metabolism and growth of infants from overweight and obese mothers
}

\author{
François-Pierre J. Martin', Sofia Moco², Ivan Montoliu³, Sebastiano Collino', Laeticia Da Silva', Serge Rezzi', Ruth Prieto4, \\ Martin Kussmann ${ }^{1,5,6}$, Jaime Inostroza ${ }^{7}$ and Philippe Steenhout ${ }^{8}$
}

BACKGROUND: The combination of maternal obesity in early pregnancy and high protein intake in infant formula feeding might predispose to obesity risk in later life.

METHODS: This study assesses the impact of breast- or formula-feeding (differing in protein content by 1.65 or $2.7 \mathrm{~g} / 100$ $\mathrm{kcal}$ ) on the metabolism of term infants from overweight and obese mothers. From birth to 3 mo of age, infants received exclusively either breast- or starter formula-feeding and until $6 \mathrm{mo}$, exclusively either a formula designed for this study or breast-feeding. From 6 to 12 mo, infants received complementary weaning food. Metabonomics was conducted on the infants' urine and stool samples collected at the age of 3, 6, and 12 mo.

RESULTS: Infant formula-feeding resulted in higher proteinderived short-chain fatty acids and amino acids in stools. Urine metabonomics revealed a relationship between bacterial processing of dietary proteins and host protein metabolism stimulated with increasing protein content in the formula. Moreover, formula-fed infants were metabolically different from breast-fed infants, at the level of lipid and energy metabolism (carnitines, ketone bodies, and Krebs cycle).

CONCLUSION: Noninvasive urine and stool metabolic monitoring of responses to early nutrition provides relevant readouts to assess nutritional requirements for infants' growth.

C oncurring with the obesity epidemic, maternal obesity is rising (1), with subsequent increased risk of childhood overweight and obesity for the offspring of overweight and obese women $(2,3)$. This increasing mother/child metabolic disease risk is driven by both genetics and pre/postnatal environmental factors (4). Since childhood overweight is a strong predictor of adult obesity, increased infant metabolic disease may translate into severe health consequences for the same subjects in adulthood $(5,6)$. Although causality is not established, rapid growth in infancy is associated with later overweight and obesity $(3,7)$. Therefore, any measure that could reduce the risk of later obesity would be of particular importance for infants born to overweight mothers, including reduction in rapid weight gain in infancy.

Optimal prenatal nutrition can reduce the risk of obesity in adults by influencing birth weight (5). Moreover, breast-feeding and introduction of complementary foods adapted to both metabolic and nutritional requirements of growing infants are also envisioned as preventive measures against obesity later in life (5). Alteration of nutrition in a critical period of development in early life can affect the subsequent pattern of growth and development of tissues and organs and may predispose individuals to several disorders later in life $(8,9)$. Interestingly, in the study conducted by Li et al. (10), breast-feeding seems to reduce the risk of being overweight during childhood in infants from obese mothers. On the other hand, obese women have difficulties in initiating and maintaining breast-feeding $(11,12)$ likely because of defects in their hormonal response to suckling $(11,12)$, birth complications, and psychological factors $(11,12)$.

For healthy mothers, breast-feeding represents the gold standard for infant health, and infant formula development aims at mimicking the physiological response to human milk intake. While protein content is essential to promote growth and brain development, protein intakes in late infancy have been found to be quite high and to exceed required intakes by large margins $(13,14)$. Moreover, high protein intake was associated with increased weight gain and higher adiposity in infancy and childhood (13,15-17). Furthermore, protein intake of breastfed infants is generally lower and may relate to a lower risk of obesity later in life $(3,18,19)$. One potential mechanism involves an enhanced stimulation of the secretion of insulin growth factor 1 (IGF-1), a trophic hormone involved in longitudinal growth as well as muscle and fat mass development (15).

Reducing protein intake during infancy, especially from 3 to 4 mo of age onwards, appears to hold promise as a measure

\footnotetext{
'Department of Molecular Biomarkers, Nestlé Institute of Health Sciences, Lausanne, Switzerland; ${ }^{2}$ Department of Natural Bioactives and Screening, Nestlé Institute of Health Sciences, Lausanne, Switzerland; ${ }^{3}$ Nestec, Nestle Research Center, Lausanne, Switzerland; ${ }^{4}$ Department of Gynecology and Obstetrics, Faculty of Medicine, University of La Frontera, Temuco, Chile; ${ }^{5}$ Faculty of Life Sciences, École Polytechnique Fédérale Lausanne (EPFL), Lausanne, Switzerland; ${ }^{6}$ Faculty of Sciences, Aarhus University, Aarhus, Denmark; ${ }^{7}$ Department of Basic Sciences, Center for Genomics and Immunological Studies (Cegin), Faculty of Medicine, University of La Frontera, Temuco, Chile; ${ }^{8}$ Nestec,

Clinical Development Unit, Lausanne, Switzerland. Correspondence: François-Pierre J. Martin (francois-pierre.martin@rd.nestle.com)
} 


\section{Articles $\mid$ Martine tal.}

for reducing the risk of later obesity. The present clinical study monitored the body weight of infants born to overweight or obese mothers split and subjected to a change in the protein content of the infant formula from the age of 3 mo onwards. More specially, the study included three feeding regimens: (i) $1.65 \mathrm{~g}$ protein $/ 100 \mathrm{kcal}$ formula, namely low-protein formula (LF); (ii) $2.7 \mathrm{~g}$ protein/100 kcal formula, namely high-protein formula (HF) that also serves as reference infant formula; and (iii) a breast-feeding (BF) control group used as reference. From birth to 3 mo of age, infants received an exclusively either breast- or starter formula-feeding, and then from 3 mo until 6 mo of age exclusively either one or the other study formula (LF or HF) or BF. From 6 to 12 mo, infants received complementary weaning food.

Thus, it becomes pertinent to deepen our knowledge not only on the human metabolism but also on gut functional ecology, during the neonatal development to understand metabolic and nutritional requirements as a function of age, growth, gut microbiota, and immune status. This lies in building knowledge not only on the metabolic deregulations but also on the effects of specific nutrients, to ultimately design novel food solutions that could improve or circumvent certain metabolic and microbial unbalances with adapted macro- and micronutrient nutritional profiles to infant needs $(20,21)$. The rapid development and increasing deployment of systems biology approaches, including metabonomics, is advancing our current understanding of physiological processes as well as food and nutrient functionalities $(22,23)$. The application of metabonomics analysis is well suited for neonatal metabolic research due to the simple, safe, and noninvasive approach that can be deployed on urine and stool samples $(21,24)$. In particular, promising metabolic insights into urine (20) and also into human breast milk (25) have led to a rapid expansion of the field of clinical applications of metabolomics in neonatology (26). Such data will serve as a reference to provide understanding of metabolic processes involved in developmental physiology, metabolic programming, and nutritional requirements.

In the present study, where the primary objective was the influence of the protein content of infant formula on body weight gain from 3 to $12 \mathrm{mo}$ of age, proton nuclear magnetic resonance ( $\left.{ }^{1} \mathrm{H} \mathrm{NMR}\right)$-based metabonomic analysis of urine and stools was used as an exploratory tool to further explore the metabolic effects of feeding during growth and development of these infants from the age of 3 to 12 mo. Metabolomics readouts were combined with body weight, body composition, and IGF data collected from infants monitored at the age of 3 , 6 , and $12 \mathrm{mo}$.

\section{RESULTS}

\section{Clinical and Anthropometric Parameters}

At the age of $3 \mathrm{mo}$, BF infants showed higher weight-for-age $Z$-scores when compared with infants who received formula (Table 1; Supplementary Figure S1 online). At the age of 6 and 12 mo, LF infants were thinner than HF or BF infants. No difference remained between $\mathrm{HF}$ and $\mathrm{BF}$ at 12 mo (Table 1; Supplementary Figure S1 and Table S3 online). All groups
Table 1. Characteristics of infants

\begin{tabular}{|c|c|c|c|}
\hline & LF & $\mathrm{HF}$ & $\mathrm{BF}$ \\
\hline \multicolumn{4}{|l|}{ Infant gender (\%) } \\
\hline Male & 55 & 51 & 51 \\
\hline Female & 45 & 49 & 49 \\
\hline Infant weight at birth $(\mathrm{kg})^{\text {a }}$ & $3.44 \pm 0.42$ & $3.57 \pm 0.42$ & $3.57 \pm 0.45$ \\
\hline Infant length at birth $(\mathrm{cm})^{\mathrm{a}}$ & $49.8 \pm 2.0$ & $50.1 \pm 1.7$ & $50.4 \pm 1.9$ \\
\hline Infant head circumference $(\mathrm{cm})^{\mathrm{a}}$ & $34.8 \pm 1.4$ & $34.9 \pm 1.3$ & $35.1 \pm 1.3$ \\
\hline Infant BMI ${ }^{\mathrm{a}}$ & $13.9 \pm 1.2$ & $14.2 \pm 1.2$ & $14.1 \pm 1.3$ \\
\hline Gestational age (mo) & $39(38-40)$ & $39(38-40)$ & $39(39-40)$ \\
\hline Weight-for-age $Z$-scores at $3 \mathrm{mo}^{\mathrm{b}}$ & $0.24 \pm 0.91$ & $0.22 \pm 0.89$ & $0.67 \pm 0.82$ \\
\hline Weight-for-age $Z$-scores at $6 \mathrm{mo}^{\mathrm{b}}$ & $0.37 \pm 0.73$ & $0.54 \pm 0.95$ & $0.89 \pm 1.03$ \\
\hline Weight-for-age $Z$-scores at $12 \mathrm{mo}^{\mathrm{b}}$ & $0.58 \pm 0.81$ & $0.88 \pm 0.88$ & $0.90 \pm 0.96$ \\
\hline
\end{tabular}

$B F$, breast-feeding; $H F$, high-protein formula; LF, low-protein formula.

atata are reported as mean $\pm S D$. ${ }^{b}$ Data are reported as median and interquartile range.

had a weight expressed in Z-score above the WHO standard growth curves references (see Supplementary Figure $S 1$ and Table S3 online). Interestingly, HF infants showed a higher IGF-1 concentration when compared with BF and LF infants at 6 mo (Table 2). No significant differences in body composition were observed.

\section{Metabonomics Analysis of Urine}

A total of 587 urine samples were acquired, from 236 infants (while only 111 infants had both urine and stool collected at the three time points). Multivariate statistical analysis of the urine metabolic profiles was conducted using orthogonal projection to latent structure discriminant analysis (O-PLS-DA) in order to assess metabolic similarities and differences between samples. O-PLS-DA revealed a significant modification of the urinary metabolic composition overtime and with the type of feeding, as noted through robust O-PLS-DA (e.g., BF: $R^{2} X=0.24, R^{2} Y=0.68, Q^{2} Y=0.39$; HF: $R^{2} X=0.22, R^{2} Y=$ $0.71, Q^{2} Y=0.43$; LF: $R^{2} X=0.27, R^{2} Y=0.48, Q^{2} Y=0.29$, see Supplementary Methods, Figure S2, and Table S4 online). Data were visualized by means of scores plot, where each point represents an individual metabolic profile. NMR, e.g., metabolic concentrations, responsible for the differences between samples in the scores plot can be extracted from the corresponding coefficients plot, where each coordinate represents a single NMR signal. The cross-validated scores plot showed statistically significant separations between the different time points of the study (see Supplementary Figure S2 online). A significant difference was noted between samples from 1-y-old infants compared with all other samples, and metabolic differences between 3 and 6 mo in BF infants were less marked than that in each formula feeding (FF) group when switching from started formula (month 3) and HF or LF (month 6). Additional O-PLS-DA analyses further highlighted strong metabolic differences between FF infants and BF infants at 3 and 6 mo of age (see Supplementary Table S4 online). The corresponding O-PLS-DA coefficients identified the specific metabolites associated with time point and group differences. 
Table 2. Clinical parameters

\begin{tabular}{lcccc}
\hline $\begin{array}{l}\text { Groups }(n \text { in } \\
\text { BF/HF/LF) }\end{array}$ & $\begin{array}{c}\text { Age in } \\
\text { months }\end{array}$ & $\begin{array}{c}\text { IGF-1 }(\mathrm{ng} / \mathrm{ml})^{\mathrm{a}} \\
(n=70 / 85 / 85)\end{array}$ & $\begin{array}{c}\text { Fat mass }(\mathrm{kg}) \\
(n=40 / 54 / 45)\end{array}$ & $\begin{array}{c}\text { Lean mass }(\mathrm{kg}) \\
(n=40 / 54 / 45)\end{array}$ \\
\hline $\begin{array}{l}\text { Breast- } \\
\text { feeding (BF) }\end{array}$ & 0 & - & - & - \\
& 3 & $80.5 \pm 51.1$ & - & - \\
& 6 & $59.1 \pm 30.6$ & - & - \\
High-protein & 0 & - & - & - \\
formula (HF) & 3 & $87.5 \pm 52.4$ & - & - \\
& 6 & $87.0 \pm 51.8^{\mathrm{b}}$ & - & - \\
& 12 & $77.5 \pm 38.1$ & $4.06 \pm 0.69$ & $6.41 \pm 0.65$ \\
Low-protein & 0 & - & - & - \\
formula (LF) & 3 & $88.1 \pm 52.1$ & - & - \\
& 6 & $76.5 \pm 60.0^{\mathrm{c}}$ & - & - \\
& 12 & $70.5 \pm 47.0$ & $4.04 \pm 0.73$ & $6.28 \pm 0.45$
\end{tabular}

$\mathrm{BF}$, breast-feeding; $\mathrm{HF}$, high-protein formula; IGF-1, insulin growth factor 1 ; LF, lowprotein formula.

aPer-protocol population. ${ }^{b}$ Difference between $\mathrm{HF}$ and $\mathrm{BF}, P=0.005$. 'Difference between $\mathrm{HF}$ and $L F, P=0.010$.

Subsequently, concentrations of influential metabolites were extracted from ${ }^{1} \mathrm{H}$ NMR spectra (see Supplementary Tables S5 and S6 online). Using metabolite set enrichment analysis (27), major changes in urinary metabolites pointed toward protein and amino acid, as well as ketogenesis and fatty acid oxidation pathways (Figure 1). Therefore, urinary metabolite excretion patterns involved in central carbon metabolism, branched-chain amino acids, fatty acid oxidation, and ketogenesis were displayed into metabolic pathways (Figure 2).

\section{Stool Metabonomics Analysis}

A total of 333 stool samples were analyzed by ${ }^{1} \mathrm{H}$ NMR spectroscopy collected from 111 infants present at the three visits and for which the urine was also available. The multivariate statistical analysis of the stool metabolic profiles revealed a significant modification of stool composition over the time, as noted by robust O-PLS-DA models (e.g., BF: $R^{2} X=0.27, R^{2} Y$ $=0.48, Q^{2} Y=0.29$; HF: $R^{2} X=0.24, R^{2} Y=0.68, Q^{2} Y=0.39$; LF: $R^{2} X=0.22, R^{2} Y=0.71, Q^{2} Y=0.43$; see Supplementary Methods, Figure S3, and Table S7 online). In each group, a significant difference was noted between samples from 1-y-old infants compared with all other samples (see Supplementary Figure S3 online). Interestingly, stool composition was not strongly changing in BF infants between 3 and 6 mo of age, whereas significant changes were noted in each FF group when switching from started formula (month 3) to HF or LF (month $6)$. Additional analyses were conducted to assess the occurrence of differences between feeding groups at each time points using O-PLS-DA (see Supplementary Table S7 online). Strong metabolic differences were observed between FF infants and BF infants at 3 and 6 mo of age, whereas no differences could be modeled when infants were 1 y old or between the HF and LF groups (see Supplementary Table S7 online). Of particular interest, a stronger metabolic change was noted in FF infants at the age of 6 mo compared with the metabolic trajectories of BF infants. The effect was similar in both HF and LF groups. Such an effect results from the shift from the starter formula to the tested HF and LF in the current study, whereas BF infants were on a constant feeding during the first months of life.

The corresponding O-PLS-DA coefficients identified the specific metabolites associated with time point and group differences. Metabolites that exhibited a concentration change over time or in response to treatment were identified for each multivariate model. Metabolite semiquantitative concentrations are reported in Table 3. Stool profiles from BF infants were marked by high concentrations of fucosylated oligosaccharides and lactic acid. FF infants showed a characteristic profile of short-chain fatty acids with higher levels of propionate, butyrate, acetate as well as 5-amino-valerate at 3 and 6 mo of age. In addition, the stool of the FF infants showed higher concentrations in free amino acids (e.g., phenylalanine, tyrosine, leucine, and isoleucine).

\section{Integration of Stool and Urine Metabonomics Data}

Integration of both stool and urine metabolic profiles across all samples ( $n=354$ for both urine and stool samples) was conducted using a PLS strategy. The generated multivariate models highlighted consistent and subtle associations between urine and stool metabolic readouts as noted by model parameters using three latent variables (e.g., $R^{2} X=0.24, R^{2} Y=0.14, Q^{2} Y=$ $0.11)$. Interpretation of the coefficients plots showed an association between fecal short-chain fatty acids (propionate and butyrate), free amino acids (phenylalanine and tyrosine), and urinary levels of creatine, glutamate, and phenylacetylglutamine.

\section{DISCUSSION}

While dietary protein content is essential for optimal growth and development of infants, high-protein intake in the first months of life has been associated with higher body fat mass and increased obesity risk (28). Despite little growth difference is reported between FF and BF infants during the first weeks of life, FF infants gain both weight and length more rapidly than BF infants from about 2 mo of age to the end of the first year of life (29). FF infants generally show a higher serum IGF-1 levelmore likely through stimulated secretion-(30,31), with inferred effects on growth and muscle and fat mass development (15). In the current study, BF infants born to overweight or obese mothers showed higher weight-for-age $Z$-scores at the age of 3 mo compared with infants fed with a starter formula. After 3 mo, weight $Z$-scores began to differ between the two FF groups, and the difference became significant at 6 mo with a higher weight in HF group. LF infants showed lower weight $Z$-scores at the ages of 6 and 12 mo compared with HF and BF infants. Surprisingly in this study, the BF group showed a significantly faster growth than the WHO Child Growth Standards. This may be explained by the fact that if the mothers were overweight or obese, it is well known that their offspring are at higher risks to become overweight or obese. The fact that infants fed LF are thinner at 12 mo of age than BF and HF-fed infants suggests that new adapted formulas with reduced energy and protein content may 


\section{Articles $\mid$ Martin et al.}

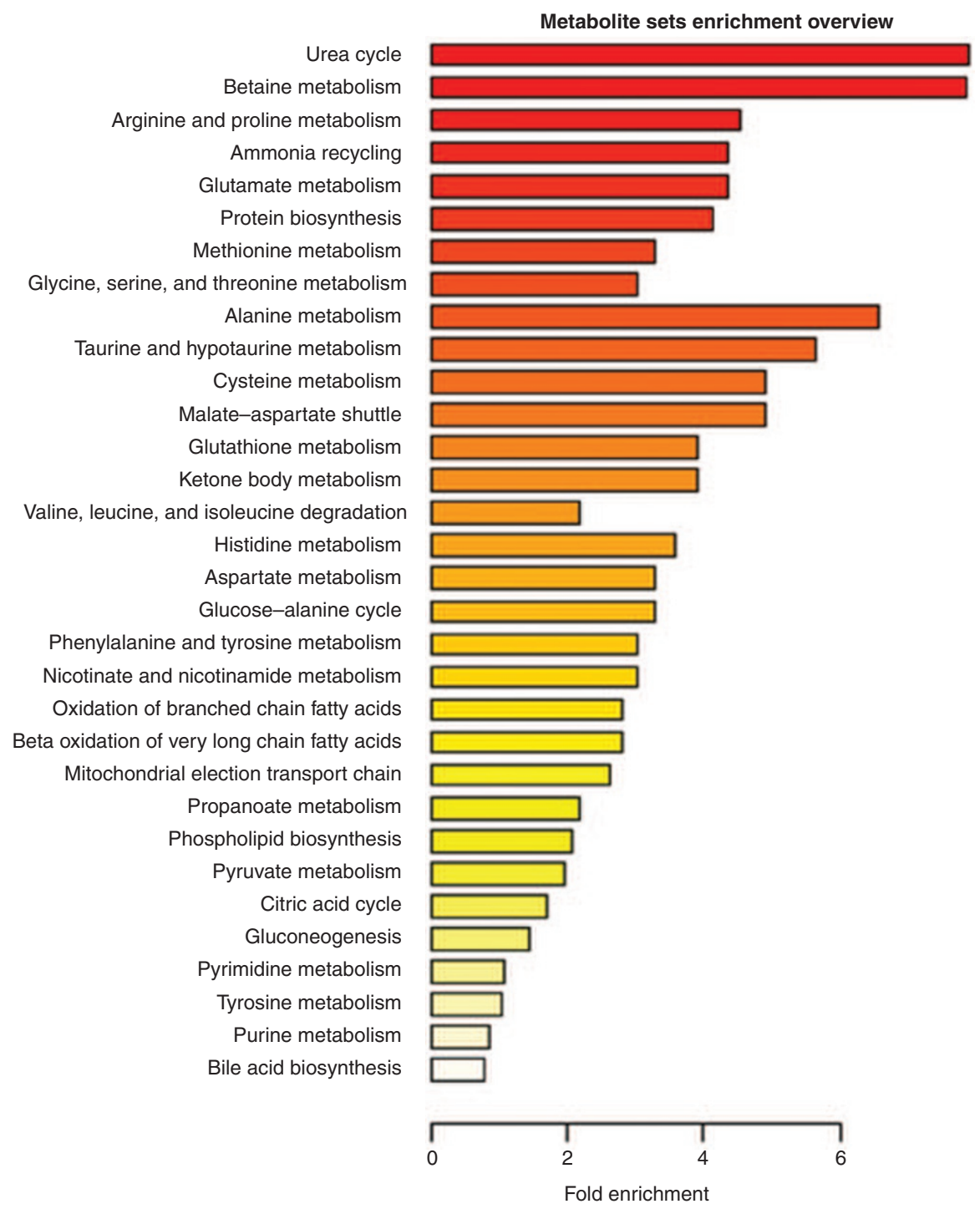

Figure 1. Summary plot for overrepresentation analysis of urinary metabolites, using metabolite set enrichment analysis. The color code of the bar plot corresponds to the calculated $P$ values (red: $P=1 \times 10^{-03}$ to white: $P=7 \times 10^{-01}$ ).

have a significant impact on reducing body weight gain in those infants born to overweight or obese mothers.

At 6 mo, infants fed with HF showed a higher concentration of IGF-1 compared with BF infants. This observation is in agreement with other reports on FF infants showing higher plasma levels of IGF-1, insulin, and certain amino acids at 4-5 mo of age (29) and with the protein intake of FF infants exceeding requirements after the first 1-2 mo of life. Taken together, all these observations may suggest that breast milk composition of overweight or obese mothers affects the growth of infants differently from the way normal-weight mother's breast milk does. In particular, during Q3-4 of the first year of life, $\mathrm{BF}$ infants are generally leaner than FF infants (29). Following introduction of complementary weaning food in our clinical trial, both BF and FF impacted neither IGF-1 nor body composition at $1 \mathrm{y}$ of age, despite lower body weight in LF infants.

One key lesson learnt from the application of metabolomics to this study is the substantial difference between the metabolic status of FF infants and BF infants, observed irrespective of the type of formula (starter, HF, and LF; Figure 3). This was marked by variations in energy recovery from the diet and infant energy metabolism.

In particular, FF infants had higher fecal concentrations of proteolytically derived short-chain fatty acids (propionate, butyrate, acetate, and 5-amino-valerate). 5-Amino-valerate is an intermediate amino acid produced from the degradation of protein hydrolysates by anaerobic bacteria, which can be further degraded to acetate, ammonia, and propionate (32). This suggests increased bacterial processing of dietary proteins (33). The higher levels of fecal free amino acids may either relate to increased proteolytic activity or excess of amino acids enriched in the formula but not absorbed in the upper gut. Not surprisingly, BF infants showed the trademark of higher fermentation of milk oligosaccharides, namely higher lactate, and an increased variety of fucosylated oligosaccharides. These metabolic signatures remain indicative of the feeding type 


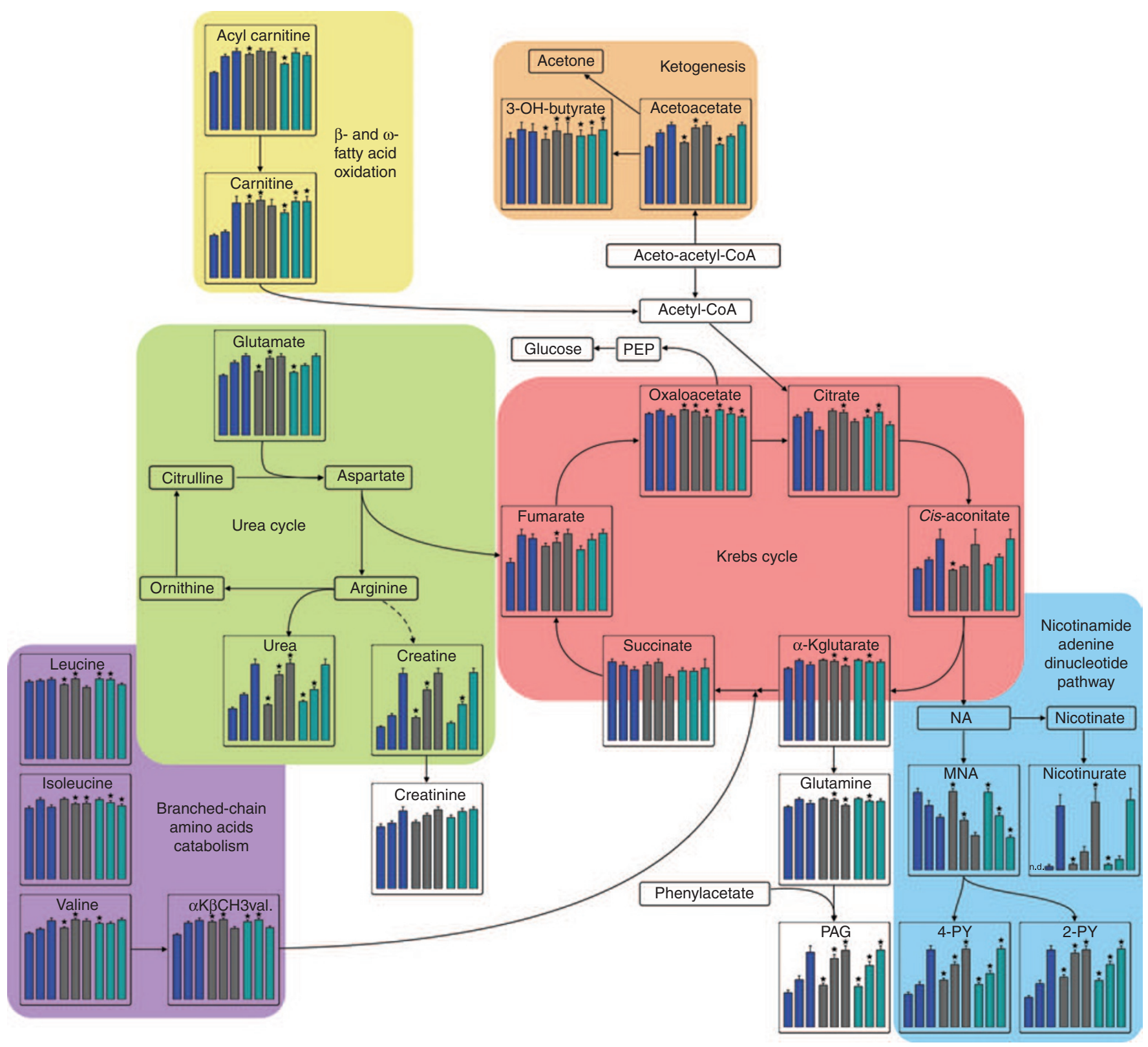

Figure 2. Metabolic pathways mapping selected urinary metabolite excretion patterns. The bar plots show mean ratio with standard error of metabolite integrals (arbitrary units) at the age of 3, 6, and 12 mo for breast-fed (BF), high-protein formula-fed (HF), and low-protein formula-fed (LF) infants (arbitrary units). The color code of the bar plot corresponds to the BF group in blue, the HF group in gray, and the LF group in green. Within each group, the first bar corresponds to semiquantitative values at $3 \mathrm{mo}$ of age, the second bar to $6 \mathrm{mo}$ of age, and the third bar to 12 mo of age. The values are given as means with standard errors. Significant differences between mean ratios of FF and BF infants were calculated with nonparametric Wilcoxon-Mann-Whitney test and are represented by ${ }^{*} P<0.05$. $\alpha$-Kglutarate, alpha-ketoglutarate; $\alpha \mathrm{K} \beta \mathrm{XH} 3$ val, $\alpha$-keto- $\beta$-methylvalerate; MNA, methylnicotinamide; NA, nicotinamide; PAG, phenylacetylglutamine; 2-PY, N1-methyl-2-pyridone-5-carboxamide; 4-PY, N1-methyl-4-pyridone-3-carboxamide; PEP, phosphoenolpyruvate.

despite introduction of complementary foods, suggesting a fundamental role of breast milk oligosaccharides in modulating digestive and bacterial metabolic activities in the first year of life.

The BF vs. FF differences in digestion and energy recovery from dietary nutrients, as observed in the stools, correlated with the differences in infant energy metabolism, as assessed by urine biochemical composition. Infants receiving FF showed an increased stimulation of the related protein metabolism, described through differences in the urea cycle and purine metabolism. The effect, observed with the starter formula at 3 mo of age, is enhanced at the age of 6 mo with HF and LF. This difference is supported through the urinary excretion pattern of phenylacetylglutamine, which is a major nitrogenous metabolite $(34,35)$. While phenylacetylglutamine production depends on the availability of phenylacetate from either host or gut microbial metabolism, its conjugation to glutamine is a key means to shuttle excess nitrogen out of the body. Its pattern closely reflects that of intermediates in the urea cycle and illustrates an alteration of the nitrogen metabolism in response 


\section{Articles $\mid$ Martin et al.}

Table 3. Influential metabolites related to amino acid and short-chain fatty acid metabolism in stool

\begin{tabular}{|c|c|c|c|c|c|c|c|c|c|}
\hline \multirow{2}{*}{$\begin{array}{l}\text { Groups } \\
\text { Age in months/ } \\
\text { metabolite level } \\
\text { (AU) }\end{array}$} & \multicolumn{3}{|c|}{ Breast-feeding } & \multicolumn{3}{|c|}{ High-protein formula } & \multicolumn{3}{|c|}{ Low-protein formula } \\
\hline & 3 & 6 & 12 & 3 & 6 & 12 & 3 & 6 & 12 \\
\hline Butyrate & $3.0 \pm 4.6$ & $8.8 \pm 8.7^{\mathrm{a}}$ & $17.2 \pm 10.1^{\mathrm{a}}$ & $10.9 \pm 9.0^{b}$ & $15.6 \pm 8.4^{\mathrm{a}, \mathrm{b}}$ & $22.9 \pm 9.6^{\mathrm{a}, \mathrm{c}}$ & $9.3 \pm 6.6^{b}$ & $13.3 \pm 9.2^{\mathrm{a}, \mathrm{c}}$ & $24.9 \pm 13.7^{\mathrm{a}, \mathrm{b}}$ \\
\hline Propionate & $1.1 \pm 2.4$ & $2.5 \pm 2.5^{a}$ & $5.5 \pm 4.2^{\mathrm{a}}$ & $4.1 \pm 3.3^{b}$ & $6.8 \pm 4.0^{\mathrm{a}, \mathrm{b}}$ & $8.3 \pm 3.9^{a, b}$ & $3.6 \pm 3.0^{b}$ & $6.6 \pm 3.4^{\mathrm{a}, \mathrm{b}}$ & $7.3 \pm 4.0^{\mathrm{a}}$ \\
\hline Acetate & $36.8 \pm 24.2$ & $59.1 \pm 31.4^{\mathrm{a}}$ & $80.2 \pm 33.2^{\mathrm{a}}$ & $76.9 \pm 37.7^{\mathrm{b}}$ & $101.5 \pm 49.3^{a, b}$ & $89.8 \pm 35.6$ & $78.7 \pm 39.9^{b}$ & $92.6 \pm 37.4^{b}$ & $87.0 \pm 29.8$ \\
\hline Lactate & $35.5 \pm 31.2$ & $35.6 \pm 29.6$ & $21.1 \pm 20.9$ & $20.9 \pm 18.5$ & $14.4 \pm 13.9^{b}$ & $10.2 \pm 8.1^{c}$ & $22.5 \pm 22.2^{c}$ & $14.5 \pm 13.6^{b}$ & $14.1 \pm 13.8$ \\
\hline Leucine & $1.6 \pm 0.8$ & $2.3 \pm 0.9^{\mathrm{a}}$ & $3.5 \pm 1.3^{\mathrm{a}}$ & $2.6 \pm 1.1^{b}$ & $3.5 \pm 1.3^{\mathrm{a}, \mathrm{b}}$ & $4.5 \pm 1.4^{\mathrm{a}, \mathrm{b}}$ & $2.9 \pm 1.1^{b}$ & $3.5 \pm 1.2^{\mathrm{a}, \mathrm{b}}$ & $4.0 \pm 1.2^{\mathrm{a}, \mathrm{c}}$ \\
\hline Isoleucine & $2.4 \pm 1.4$ & $3.3 \pm 1.4^{\mathrm{a}}$ & $5.4 \pm 2.3^{\mathrm{a}}$ & $3.6 \pm 1.7^{b}$ & $5.0 \pm 2.2^{\mathrm{a}, \mathrm{b}}$ & $6.9 \pm 2.4^{\mathrm{a}, \mathrm{b}}$ & $4.2 \pm 1.6^{\mathrm{b}}$ & $5.2 \pm 1.9^{a, b}$ & $6.4 \pm 2.1^{\mathrm{a}, \mathrm{c}}$ \\
\hline Valine & $4.0 \pm 2.9$ & $5.2 \pm 2.3$ & $7.3 \pm 2.8^{\mathrm{a}}$ & $4.9 \pm 2.3^{b}$ & $6.7 \pm 3.1^{\mathrm{a}, \mathrm{c}}$ & $8.7 \pm 3.0^{\mathrm{a}, \mathrm{c}}$ & $5.6 \pm 2.0^{\mathrm{a}}$ & $6.7 \pm 2.3^{a, c}$ & $7.9 \pm 2.5^{\mathrm{a}}$ \\
\hline
\end{tabular}

No statistical differences were found between FF groups.

$\mathrm{AU}$, arbitrary units; FF, formula feeding.

aMetabolite values at 6 and 12 mo compared with values at 3 mo are statistically different at a 95\% confidence interval in each group. b.c Differences with breast-feeding group at the same age is statistically different at 99 and $95 \%$ confidence interval, respectively.

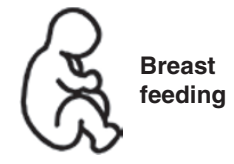

Carbohydrate metabolism

$\uparrow$ Lactate (stool)

$\uparrow$ Milk oligosaccharides

(stool)

\section{Energy metabolism \\ Different Krebs cycle and \\ NAD/NADP metabolic \\ pathways}

Lipid metabolism

Ketogenesis (milk lipids)

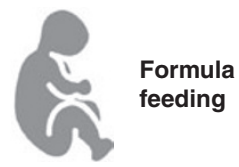

Growth and development 个 IGF-1

Protein metabolism

$\uparrow$ Protein-derived SCFAs

(stool)

$\uparrow$ Urea cycle and nitrogen

balance

\section{Lipid metabolism \\ $\uparrow \beta$-oxidation (carnitine) \\ $\uparrow$ Ketogenesis}

(lipids and ketogenic AAs)

Figure 3. Scheme summarizing main metabolic differences between breast-fed and formula-fed infants. AAs, amino acids; IGF-1, insulin growth factor 1; SCFAs, short-chain fatty acids.

to the type of feeding, HF resulting in the highest increase in nitrogen changes.

Furthermore, urine analysis revealed that major differences in lipid metabolism were observed at the age of 3 mo between $\mathrm{BF}$ and $\mathrm{FF}$ infants, which varied further according to the type of infant formula. The concentrations of carnitine and acyl carnitine tend to be higher in FF infants, which may result from the standard supplementation of formula with free carnitine. Unfortunately, we do not have measurement of the carnitine content in the human milk of the BF group and cannot verify this hypothesis. Since fatty acid $\beta$-oxidation involves carnitinedependent enzyme activities, formula supplementation may avoid its saturation and enables a greater lipid mobilization $(36,37)$. FF infants also revealed a more sustained production of acyl carnitine acids throughout the first year of life, whereas

BF infants showed a time-dependent metabolic adaptation. Fat may be underused as an energy substrate due to the inhibition of mitochondrial $\beta$-oxidation, for instance, when there is not enough carnitine available $(36,37)$. Breast milk composition is well known to change overtime, with a decreasing content in protein and increased proportion of fat (38). Therefore, BF infants may exhibit here a specific metabolic adaptation to breast milk compositional changes involving compensatory mechanisms for metabolizing fatty acids.

A time-dependent ketogenic metabolic signature was highlighted in parallel to these metabolic variations in energy metabolism. Two ketone bodies, namely, 3-D-hydroxybutyrate and acetoacetate, were increasingly excreted in urine during growth of all infants. Ketogenesis is generally stimulated when fatty acid $\beta$-oxidation and production of acetylcoenzyme A supersedes the processing capacity of the Krebs cycle. In BF infants, the metabolic adaptation to milk fat may therefore lead to ketogenesis during the first year of life. Since FF infants show a similar response, the signature may describe a generally adapted metabolism to handle dietary fat for energy production. However, differences exist between $\mathrm{FF}$ and $\mathrm{BF}$ infants. Whereas 3-D-hydroxybutyrate remains at lower concentration with FF, the acetoacetate level was higher, which suggests a different contribution from protein and lipid metabolism to ketogenesis according to the type of feeding. On one hand, 3-D-hydroxybutyrate can be produced by gluconeogenesis, mainly from gluconeogenic amino acids (mainly valine), and be further oxidized to acetoacetate. On the other hand, acetoacetate can also be generated from ketogenetic amino acids (mainly leucine and lysine) and converted back to 3-D-hydroxybutyrate. Since, at 6 mo of age, the acetoacetate level tends to be higher with HF, FF may stimulate ketogenesis through its specific ketogenic protein/amino acid content. Moreover, the lower level of 3-D-hydroxybutyrate with $\mathrm{FF}$ and the absence of dose-dependent response to the protein 
content in FF suggest other differences in hepatic gluconeogenesis between $\mathrm{BF}$ and $\mathrm{FF}$.

The overall changes in energy production from protein and lipid metabolism result in different and complex fluxes through Krebs cycle and nicotinamide adenine dinucleotide/nicotinamide adenine dinucleotide phosphate metabolic pathways. In particular, the concomitant patterns in $\mathrm{N}$-methylnicotinamide and 2-PY and 4-PY (its two direct catabolic products) illustrate different biochemical cascades, with inferred relationships on energy production and use by newborn metabolism. Interestingly, the introduction of complementary food activates another branch in the nicotinamide adenine dinucleotide/nicotinamide adenine dinucleotide phosphate pathway as noted by significantly increased urinary excretion of nicotinurate. Overall, this provides an additional example on how increased diversity in macro- and micronutrients leads to coordinated metabolic adaptative process for energy production and use by infant metabolism.

\section{Conclusion}

Formula development aims at mimicking the physiological response to human breast milk intake. The present study shows how noninvasive sampling of urine and stool followed by metabolomic profiling can monitor the metabolic response and the requirements of infants during growth. Such metabolic profiles provide biological readouts and insights on both host and bacterial metabolism during growth and development of infants, by means of which we can assess the degree of metabolic equivalence between $\mathrm{BF}$ and FF. We demonstrate a significant difference between the metabolic status of FF and $\mathrm{BF}$ infants, and this difference was even preserved irrespective of the type of tested formula (starter, high-protein, or low-protein formula). A specific metabolic signature associated with increasing protein content in the formula was observed in FF infants, which were still metabolically very different from BF infants. We have attempted to interpret the observed biochemical changes in terms of metabolic regulation and adaptation to feeding; however, we are aware that more detailed studies will have to be undertaken to fully unravel infants' metabolism in a dynamic fashion. We believe that further developments of such systems biology-oriented omic approaches in neonatology will deliver novel nutritional and metabolic hypotheses that can be targeted with new personalized infant feeding concepts.

\section{METHODS}

\section{Experimental Design}

This clinical study was approved by the Ethical Committee of the Universidad de la Frontera, Temuco, Chile, and conducted by Jaime Inostroza and colleagues. The clinical trial is registered at ClinicalTrials.gov with the identifier NCT00820833. The study is a double-blind, controlled, randomized, single-site clinical trial of two parallel FF groups with a nonblinded BF group (Figure 4). In each FF group, 64 infants completed the study, while a total of $56 \mathrm{BF}$ infants (nonrandomized) were enrolled as a reference group. Subjects who were $\mathrm{FF}$ were randomly assigned to one of the two treatment groups starting at the age of $3 \mathrm{mo}$, namely, a low-protein and low caloric density formula with probiotics (LF; $1.65 \mathrm{~g}$ protein $/ 100 \mathrm{kcal}$, with a caloric density of $64 \mathrm{kcal} / \mathrm{dl}$ and the probiotics Bifidobacterium lactis CNCM I-3446 and Lactobacillus rhamnosus LPR CGMCC 1.3724 with a target concentration for both probiotics of $2 \times 10^{07}$ colonyforming unit/g) or a high-protein formula (HF; $2.7 \mathrm{~g}$ protein $/ 100$ $\mathrm{kcal}$, with a caloric density of $65.6 \mathrm{kcal} / \mathrm{dl}$ without probiotics). See Supplementary Table S1 for more information on tested formulas. From birth to 3 mo of age, infants received an exclusive breast- or starter formula-feeding (1.8 g protein/100 kcal); from 3 to 6 mo, study formula or breast-feeding; and from 6 to 12 mo, mixed feeding. The

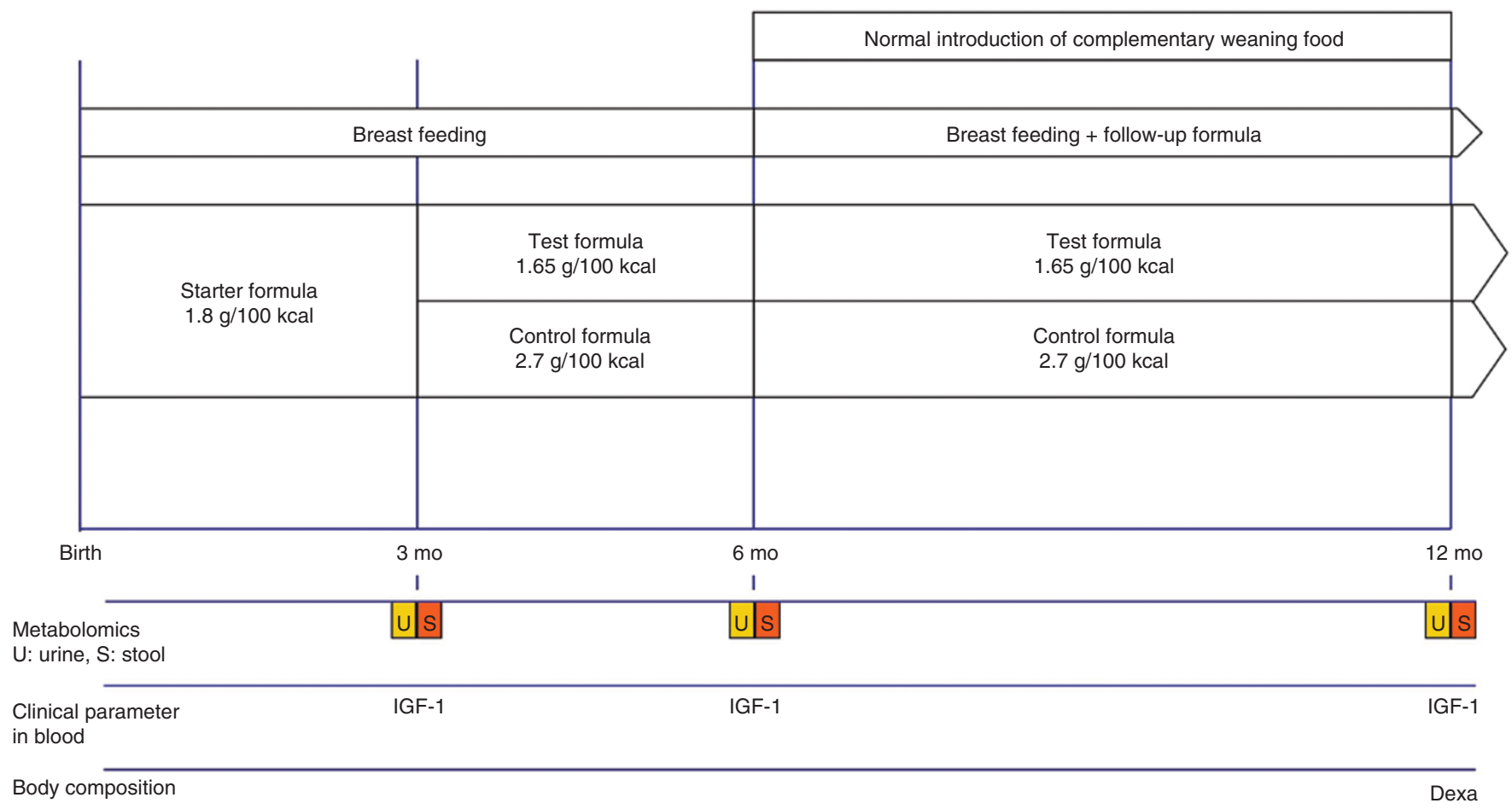

Figure 4. Experimental design. IGF-1, insulin growth factor 1. 
follow-up formula that the $\mathrm{BF}$ group can receive after the sixth month of exclusive breast-feeding consists of $2.4 \mathrm{~g}$ protein $/ 100 \mathrm{kcal}$, with a caloric density of $67 \mathrm{kcal} / \mathrm{dl}$.

\section{Participants}

All participants were healthy newborn infants of mothers with a BMI between 26 and $35 \mathrm{~kg} / \mathrm{m}^{2}$, as calculated based on the height and the prepregnancy weight at the first pregnancy care visit to the health care center (i.e., before the 13th wk of pregnancy; see Supplementary Table S2 online). All infants were preenrolled before birth after having obtained his/her legal representative's informed consent. The babies were full term $(37 \geq$ wk gestation $\leq 42)$, with a birth weight between 2,500 and 4,800 g, and born from a singleton birth. The following exclusion criteria were employed: babies with a body weight $\leq 5$ th percentile for the corresponding gestational age, newborns whose mothers have type 1 or type 2 diabetes, smoking more than five cigarettes per day during pregnancy, mothers with a drug dependence during pregnancy, or those with a chronic infectious disease. Pregnancies marked by a congenital illness or malformation that may affect normal growth, or with other significant prenatal and/or postnatal disease were also excluded, as well as babies undergoing a rehospitalization for more than $2 \mathrm{~d}$ in the first $14 \mathrm{~d}$ of life, except for jaundice.

\section{Sample Collection}

Urine, blood, and stool samples were collected at the age of 3, 6, and 12 mo. Urine samples $(1 \mathrm{ml})$ were collected by means of a sterile plastic labeled bag and were stored at $-40^{\circ} \mathrm{C}$, prior to analysis. Stool samples $(\sim 10 \mathrm{~g})$ were collected using a plastic labeled bag and stored at $-80^{\circ} \mathrm{C}$. Blood $(1 \mathrm{ml})$ was collected before feeding, in Heparin lithium tubes, centrifuged at $4^{\circ} \mathrm{C}$ and kept on ice. Plasma $(\sim 150 \mu \mathrm{l})$ was transferred to a labeled cryostat tube and kept at $-80^{\circ} \mathrm{C}$.

\section{Clinical and Anthropometric Measures}

Infants were weighed without clothing or nappy/diaper on electronic weighing scales. The same scales were used for all infants at all visits. Scales were calibrated as per manufacturer's recommendations at the start of the study and every month thereafter until the end of the study. Body composition measurements at 12-13 mo were performed using a DXA Lunar Prodigy Advance with software EnCore version 13.6 (GE Health Care, Madison, WI). Determination of IGF-I was performed by ELISA using kit from ALPCO (ALPCO Diagnostics, Salem, NH).

\section{Metabonomics Analysis}

Two hundred microliter of urine and $80 \mathrm{mg}$ of wet stool from the collected samples were freeze-dried. The dry extracts were solubilized in $60 \mu \mathrm{l}$ (urine) and $1 \mathrm{ml}$ (stool) deuterated phosphate buffer solution $0.2 \mathrm{~mol} / 1 \mathrm{KH}_{2} \mathrm{PO}_{4}$, containing $0.3 \mathrm{~m} \mathrm{~mol} / \mathrm{l}$ of sodium azide as antibacterial agent, and $1 \mathrm{~m} \mathrm{~mol} / \mathrm{l}$ of sodium 3-(trimethylsilyl)$\left[2,2,3,3-2 \mathrm{H}_{4}\right]-1$-propionate (TSP, chemical shift reference $\delta_{\mathrm{H}}=0.0$ $\mathrm{ppm}$ ). The homogenates were centrifuged at $17,000 \mathrm{~g}$ for $10 \mathrm{~min}$, and $60 \mu \mathrm{l}$ of the supernatant were transferred into $1.7 \mathrm{~mm}$ NMR tubes. ${ }^{1} \mathrm{H}$ NMR metabolic profiles were acquired with a Bruker Avance II 600 $\mathrm{MHz}$ spectrometer equipped with a $1.7 \mathrm{~mm}$ probe at $300 \mathrm{~K}$ (Bruker Biospin, Rheinstetten, Germany), using a standard pulse sequence with water suppression and processed using TOPSPIN (version 2.1, Bruker, Germany) software package as reported previously (39).

\section{Statistical Analysis}

Data processing and analysis was conducted as reported previously $(33,40)$. Briefly, chemometric analysis was performed using SIMCA-P+ (version 11.5, Umetrics AB, Umeå, Sweden) and in-house developed MATLAB routines (see Supplementary Methods online). Principal component analysis (41), PLS, and orthogonal projection to latent structures (42) were used with a sevenfold cross-validation to assess the validity of the model. The multivariate pattern recognition techniques used in this study were based on O-PLS approach with unit-variance scaling. In the O-PLS-DA algorithm, the variation in X (NMR metabolic profiles) is decomposed in three parts: first, the variation in $\mathrm{X}$ (metabolic profiles) related to $\mathrm{Y}$ (group, time), and the two last parts contain the specific systemic variation in $\mathrm{X}$ and residual, respectively. This leads to a model with a minimal number of predictive components defined by the number of degrees of freedom between group variances. Influential metabolites identified from the multivariate data analysis were relatively quantified by signal integration and analyzed using Wilcoxon-Mann-Whitney tests. Due to the exploratory nature of the work and their identification by multivariate data analysis (on group-time interactions, group, and time differences), the reported $P$ values were not corrected for multiple testing. Metabolic pathway analysis was conducted by submitting the list of influential metabolites obtained through multivariate data analysis to metabolite set enrichment analysis using the web-based interface MetaboAnalyst 2.0 (27).

Anthropometric data are presented as Z-scores for weight from 3 to 12 mo and calculated based on the WHO 2006 Child Growth Standards (Table 1; Supplementary Figure S1 and Table S3 online) (43). Z-scores are expressed as means and SD. Data are presented without any correction for intervention baseline at 3 mo.

\section{SUPPLEMENTARY MATERIAL}

Supplementary material is linked to the online version of the paper at http://www.nature.com/pr

\section{ACKNOWLEDGMENTS}

We thank S. Colombo Mottaz and F. Chauffard for clinical trial management, D. Grathwohl and D. Egli for their scientific input and discussion in relation to the infant growth and development, and D. Barron for managerial support at NIHS to S.M.

\section{REFERENCES}

1. Sebire NJ, Jolly M, Harris JP, et al. Maternal obesity and pregnancy outcome: a study of 287,213 pregnancies in London. Int J Obes Relat Metab Disord 2001;25:1175-82.

2. Whitaker RC. Predicting preschooler obesity at birth: the role of maternal obesity in early pregnancy. Pediatrics 2004;114:e29-36.

3. Weng SF, Redsell SA, Swift JA, Yang M, Glazebrook CP. Systematic review and meta-analyses of risk factors for childhood overweight identifiable during infancy. Arch Dis Child 2012;97:1019-26.

4. Sewell MF, Huston-Presley L, Super DM, Catalano P. Increased neonatal fat mass, not lean body mass, is associated with maternal obesity. Am J Obstet Gynecol 2006;195:1100-3.

5. Yang Z, Huffman SL. Nutrition in pregnancy and early childhood and associations with obesity in developing countries. Matern Child Nutr 2013;9:Suppl 1:105-19.

6. Whitaker RC, Wright JA, Pepe MS, Seidel KD, Dietz WH. Predicting obesity in young adulthood from childhood and parental obesity. $\mathrm{N}$ Engl J Med 1997;337:869-73.

7. Adair LS, Fall CH, Osmond C, et al.; COHORTS group. Associations of linear growth and relative weight gain during early life with adult health and human capital in countries of low and middle income: findings from five birth cohort studies. Lancet 2013;382:525-34.

8. McMillen IC, Robinson JS. Developmental origins of the metabolic syndrome: prediction, plasticity, and programming. Physiol Rev 2005;85:571633.

9. Koletzko B, Aggett PJ, Bindels JG, et al. Growth, development and differentiation: a functional food science approach. Br J Nutr 1998;80:Suppl 1:S5-45.

10. Li C, Kaur H, Choi WS, Huang TT, Lee RE, Ahluwalia JS. Additive interactions of maternal prepregnancy BMI and breast-feeding on childhood overweight. Obes Res 2005;13:362-71.

11. Rasmussen KM, Hilson JA, Kjolhede CL. Obesity as a risk factor for failure to initiate and sustain lactation. Adv Exp Med Biol 2002;503:217-22.

12. Rasmussen KM, Kjolhede CL. Prepregnant overweight and obesity diminish the prolactin response to suckling in the first week postpartum. Pediatrics 2004;113:e465-71.

13. Ohlund I, Hernell O, Hörnell A, Stenlund H, Lind T. BMI at 4 years of age is associated with previous and current protein intake and with paternal BMI. Eur J Clin Nutr 2010;64:138-45.

14. Butte NF, Fox MK, Briefel RR, et al. Nutrient intakes of US infants, toddlers, and preschoolers meet or exceed dietary reference intakes. J Am Diet Assoc 2010;110:Suppl 12:S27-37. 
15. Rolland-Cachera MF, Deheeger M, Akrout M, Bellisle F. Influence of macronutrients on adiposity development: a follow up study of nutrition and growth from 10 months to 8 years of age. Int J Obes Relat Metab Disord 1995;19:573-8.

16. Singhal A, Kennedy K, Lanigan J, et al. Nutrition in infancy and long-term risk of obesity: evidence from 2 randomized controlled trials. Am J Clin Nutr 2010;92:1133-44.

17. Koletzko B, von Kries R, Closa R, et al.; European Childhood Obesity Trial Study Group. Lower protein in infant formula is associated with lower weight up to age 2 y: a randomized clinical trial. Am J Clin Nutr 2009;89:1836-45.

18. Arenz S, Rückerl R, Koletzko B, von Kries R. Breast-feeding and childhood obesity-a systematic review. Int J Obes Relat Metab Disord 2004;28:1247-56.

19. Harder T, Bergmann R, Kallischnigg G, Plagemann A. Duration of breastfeeding and risk of overweight: a meta-analysis. Am J Epidemiol 2005;162:397-403.

20. Fanos V, Antonucci R, Barberini L, Atzori L. Urinary metabolomics in newborns and infants. Adv Clin Chem 2012;58:193-223.

21. Moco S, Collino S, Rezzi S, Martin FP. Metabolomics perspectives in pediatric research. Pediatr Res 2013;73(4 Pt 2):570-6.

22. Nicholson JK. Global systems biology, personalized medicine and molecular epidemiology. Mol Syst Biol 2006;2:52.

23. Martin FP, Collino S, Rezzi S, Kochhar S. Metabolomic applications to decipher gut microbial metabolic influence in health and disease. Front Physiol 2012;3:113.

24. Antonucci R, Atzori L, Barberini L, Fanos V. Metabolomics: the "new clinical chemistry" for personalized neonatal medicine. Minerva Pediatr 2010;62:3 Suppl 1:145-8.

25. Marincola FC, Noto A, Caboni P, et al. A metabolomic study of preterm human and formula milk by high resolution NMR and GC/MS analysis: preliminary results. J Matern Fetal Neonatal Med 2012;25:Suppl 5:62-7.

26. Fanos V, Iacovidou N, Puddu M, Ottonello G, Noto A, Atzori L. Metabolomics in neonatal life. Early Hum Dev 2013;89:Suppl 1:S7-10.

27. Xia J, Mandal R, Sinelnikov IV, Broadhurst D, Wishart DS. MetaboAnalyst 2.0-a comprehensive server for metabolomic data analysis. Nucleic Acids Res 2012;40:W127-33.

28. Koletzko B. Long-term consequences of early feeding on later obesity risk. Nestle Nutr Workshop Ser Pediatr Program 2006;58:1-18.
29. Ziegler EE. Growth of breast-fed and formula-fed infants. Nestle Nutr Workshop Ser Pediatr Program 2006;58:51-9; discussion 59-63.

30. Savino F, Fissore MF, Grassino EC, Nanni GE, Oggero R, Silvestro L. Ghrelin, leptin and IGF-I levels in breast-fed and formula-fed infants in the first years of life. Acta Paediatr 2005;94:531-7.

31. Savino F, Nanni GE, Maccario S, Oggero R, Mussa GC. Relationships between IGF-I and weight Z score, BMI, tricipital skin-fold thickness, type of feeding in healthy infants in the first 5 months of life. Ann Nutr Metab 2005;49:83-7.

32. Barker HA. Pathway of 5-aminovalerate degradation by Clostridium aminovalericum. Curr Top Cell Regul 1985;26:349-54.

33. Martin FP, Sprenger N, Montoliu I, Rezzi S, Kochhar S, Nicholson JK. Dietary modulation of gut functional ecology studied by fecal metabonomics. J Proteome Res 2010;9:5284-95.

34. Brusilow SW. Phenylacetylglutamine may replace urea as a vehicle for waste nitrogen excretion. Pediatr Res 1991;29:147-50.

35. Mokhtarani M, Diaz GA, Rhead W, et al. Urinary phenylacetylglutamine as dosing biomarker for patients with urea cycle disorders. Mol Genet Metab 2012;107:308-14.

36. Schiff D, Chan G, Seccombe D, Hahn P. Plasma carnitine levels during intravenous feeding of the neonate. J Pediatr 1979;95:1043-6.

37. Olson AL, Nelson SE, Rebouche CJ. Low carnitine intake and altered lipid metabolism in infants. Am J Clin Nutr 1989;49:624-8.

38. Ballard O, Morrow AL. Human milk composition: nutrients and bioactive factors. Pediatr Clin North Am 2013;60:49-74.

39. Baur P, Martin FP, Gruber L, et al. Metabolic phenotyping of the Crohn's disease-like IBD etiopathology in the TNF( $\triangle \mathrm{ARE} / \mathrm{WT})$ mouse model. J Proteome Res 2011;10:5523-35.

40. Rezzi S, Martin FP, Alonso C, et al. Metabotyping of biofluids reveals stress-based differences in gut permeability in healthy individuals. J Proteome Res 2009;8:4799-809.

41. Wold S, Esbensen K, Geladi P. Principal Component Analysis. Chemom Intell Lab Syst 1987;2:37-52.

42. Trygg J, Wold S. O2-PLS, a two-block (X-Y) latent variable regression (LVR) method with an integrated OSC filter. J Chemom 2003;17:53-64.

43. WHO Child Growth Standards. http://www.who.int/childgrowth/en. 2006. 